\title{
The clinical trials system is broken
}

\author{
Vincent T DeVita Jr
}

The Co-Directors of the Center for Management Research in Healthcare at Vanderbilt, David Dilts and Alan Sandler, reported a study of an alarming nature to the National Cancer Institute (NCl). The study was covered by The Cancer Letter and documented that cancer trials take an average of 800 days to start. The clinical trials program in the US is broken and apparently nobody has noticed: 800 days! Can you imagine that? The response of the $\mathrm{NCl}$ Director was a vow to cut the time to approval in half. At 400 days the system would still be broken. Dilts and Sandler reported that for cooperative-group studies, a protocol takes 800 days from conception to activation, with an additional 200 days at a cancer center. Even an investigator-initiated trial at a cancer center takes a median of 116 to 252 days, and if you make amendments to a study it goes back to the end of the line. Furthermore, the study by Dilts and Sandler was conducted by two cooperative groups at four centers selected by $\mathrm{NCl}$ because they scored better than most (in peer review) on the function of their clinical trials programs. The solutions offered by the authors of the study to rectify this situation were not of much help (e.g. start fewer studies, stop tweaking studies).

We live in an age where we are blessed with bounteous information about critical steps in the pathways that cancer cells use to outstrip the growth of normal cells and information about how their cell-death pathways are broken. Although we have the specific agents and the opportunity to capitalize on the whole concept of 'targeted therapy' we find ourselves unable to use these advances. If one looks at past successes in treatment, they were

\section{At my own \\ institution I \\ reside on many \\ committees \\ involved in \\ protocol \\ review, and II \\ can say with \\ authority \\ that we are \\ hopelessly \\ over-regulated}

VT DeVita Jr is the Editor-in-Chief of Nature Clinical Practice Oncology.

Competing interests

The author declared no competing interests.

www.nature.com/clinicalpractice doi:10.1038/ncponc1263 characterized by the ability to test an idea rapidly, and to tweak studies to allow swift adjustment of protocols in response to events happening in real time. I have not examined the protocols in question directly, but I would not hesitate to suggest that a study that takes 800 days to activate is hopelessly outdated the day it starts. Furthermore, if investigators are encouraged to avoid tweaking, then the talents of our best investigators are either not being used or are being wasted.

At my own institution I reside on many committees involved in protocol review, and I can say with authority that we are hopelessly over-regulated. Although cancer centers are not perfect and have many problems of their own, they are still where the knowledge base is. The requisite talent to know the right way to design and modify an ongoing study does not reside on remote review committees at the $\mathrm{NCl}$ or the FDA, yet those are the places where the delays are greatest. Too many cooks are spoiling the broth.

A step towards fixing this problem would be to delegate the entire review and approval process, at least for phase I and II studies, to $\mathrm{NCl}$-designated cancer centers with $\mathrm{NCl}$ approved clinical trials programs, with the $\mathrm{NCl}$ and FDA only retaining audit responsibilities. This was the role envisioned for cancer centers by those who framed the $\mathrm{NCl}$ Act of 1971 . Most of these regulations have been imposed on us by the US congress in the name of patients' safety. Review boards set up and codified in regulations to protect patients are doing just the opposite. They prevent patients from having access to the fruits of the war on cancer and the best we have to offer. 